\title{
VALORACIONES SOBRE EL CLAUSTRO DEL PROFESORADO DE EDUCACIÓN SECUNDARIA OBLIGATORIA Y DE BACHILLERATO
}

\author{
ASSESMENTS ON TEACHING STAFF \\ OF SECONDARY EDUCATION
}

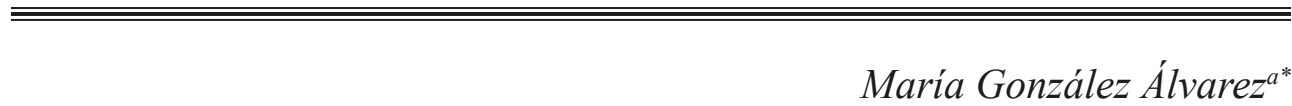

Fechas de recepción y aceptación: 21 de marzo de 2020 y 28 de abril de 2020

DOI: https://doi.org/10.46583/edetania_2021.59.730

Resumen: En el presente trabajo se pretende realizar un estudio exploratorio sobre el claustro del profesorado de los centros de ESO y Bachillerato. Los objetivos que se proponen se refieren a la formación inicial y permanente recibida por el profesorado respecto al claustro, la valoración de la importancia de sus competencias y el cumplimiento de estas, el claustro como órgano de participación, su eficacia y la atención que le prestan las administraciones educativas. Se elaboró y puso a disposición del profesorado un protocolo específico que fue enviado por correo electrónico a una muestra de centros seleccionados al azar en función de su titularidad y de la población de las distintas provincias de España. Respondieron a este 1318 profesores y profesoras. Se realizaron los correspondientes análisis descriptivos, diferencia de medias y análisis multivariado. El coeficiente de fiabilidad de Cronbach es de .96. Del análisis se colige que el profesorado otorga una alta valoración a la importancia de las competencias del claustro para la educación del alumnado y el funcionamiento del centro, por lo que se encuentra satisfecho como miembro de este y se considera un órgano razonablemente eficaz. El profesorado entiende que debe mejorarse su formación inicial y permanente respecto a los temas referidos a este órgano, así como el grado de cumplimiento de sus competencias, la

${ }^{a}$ Departamento de Personalidad, Evaluación y Tratamiento Psicológico I (Personalidad, Evaluación y Psicología Clínica). Universidad Complutense de Madrid.

*Correspondencia: Universidad Complutense de Madrid. Facultad de Psicología. Avenida Séneca, 2. 28040 Madrid. España.

E-mail: maria_gonzalez@ucm.es 
valoración que las administraciones educativas realizan de su funcionamiento y la atención que prestan a sus acuerdos.

Palabras clave: educación secundaria, formación inicial, formación permanente, competencias del claustro, claustro escolar.

Abstract: This paper aims to conduct an exploratory study about the teaching staff in secondary education schools. The goals proposed are referred to the initial and ongoing training of teachers concerning the teaching staff, the value given to the competences and their accomplishment, the board as a participative body, its effectiveness and the attention paid to the board by the educative administration. To achieve this goal, we have written a specific test protocol that has been sent by email to schools chosen at random considering the ownership of the schools and the population of the different provinces of Spain. The protocol was answered by 1318 teachers. We have carried out the appropriate descriptive analysis, media differences and multivariable analysis. Cronbach's coefficient of reliability is 0.96 . The analysis shows that teachers highly value the importance of the teaching staff competences for the education of the students and the school management, so they are also satisfied as members of the teaching staff, considering it to be an effective body for the school. Teachers consider that their initial and ongoing training in the board-related aspects should improve, as well as the accomplishment of the general board's tasks, the value given to the board coordinated by the administration and the attention it pays to its agreements and suggestions.

Keywords: secondary education, initial training, ongoing training, teaching staff competences, teaching staff.

\section{INTRODUCCIÓN}

En el artículo 27.7 de la Constitución española de 1978 se consagra el derecho del profesorado, familias y alumnado a intervenir en el control y gestión de los centros sostenidos por la Administración con fondos públicos. Derecho que se ha ido desarrollando mediante las correspondientes leyes orgánicas y las específicas de las distintas comunidades autónomas en el ámbito de sus competencias.

En el presente trabajo se pretende analizar el claustro de los centros de educación secundaria obligatoria (en adelante ESO) y bachillerato, órgano específico de participación del profesorado. Es un tema escasamente tratado en la investigación educativa a pesar de su importancia para el funcionamiento del centro y la enseñanza del alumnado. No obstante, tiene una atención central en el desarrollo y quehacer diario de los centros educativos, fundamentalmente en lo que se refiere a aspectos como el reparto de competencias con el con- 
sejo escolar y al debate entre dirección y profesorado por asumir las distintas atribuciones-preeminencia en el claustro. Con este objetivo se analiza la valoración que se otorga a la formación inicial y permanente que se recibe en relación con el claustro así como los distintos temas que pueden incluirse en sus programas de formación; la valoración que se realiza de las competencias que la normativa asigna al órgano y cómo las cumple en su funcionamiento; la duración media de sus sesiones de trabajo y el número que se realizan durante el curso; la importancia que tiene como órgano de participación del profesorado y su eficacia; cómo atiende a los problemas reales del centro; cómo se considera que las administraciones valoran su funcionamiento y atienden a los acuerdos y sugerencias que realizan.

La participación en educación se entiende como un factor necesario en los centros para el desarrollo del alumnado y el éxito escolar (Andrés-Cabello y Giró-Miranda, 2016; Bolívar, 2006), debiendo considerarse el ejercicio de dicha participación como algo más que un formalismo (Moliner-García, TraverMartí, Ruiz-Bernardo y Segarra-Arnau, 2016). Según Martín-Bris y MuñozMartínez (2010) sin la participación del profesorado en los procesos de cambio educativo no existen posibilidades de que este tenga éxito. Participación que para Raso-Sánchez, Sola-Martínez e Hinojo-Lucena (2017) es bien valorada por el $92,8 \%$, estando las profesoras más satisfechas que sus colegas varones respecto a la participación en relación con los aspectos organizativos del centro.

Se entiende que tanto en la formación inicial como permanente deben incluirse aspectos relativos al claustro, al estar la calidad de la enseñanza relacionada con la formación del profesorado (Barber y Mourshed, 2007; More y MulaBenavent, 2015; OCDE, 2014; Rivas-Borrel, 2014; Sanz-Ponce, Hernando-More y Mula-Benavent, 2015; Tiana-Ferrer, 2011). Según Ordoñez-Sierra (2008) el profesorado tiene importantes carencias en su formación inicial respecto al conocimiento y participación en las estructuras participativas del centro.

Señalan Sanz-Ponce et al. (2015) que "la formación del profesorado de Educación Secundaria se ha enfrentado tradicionalmente a diversos retos/ problemas (...) teniendo que elegir entre un carácter formativo o instructivo" (2015, p. 217).

Ros-Garrido y García-Rubio (2016) señalan la conveniencia de que el profesorado lleve a las aulas las reformas educativas, para lo cual son necesarias tanto una adecuada formación inicial como permanente. En relación con la 
formación inicial, en materia de igualdad de género, Pinedo-González, ArroyoGonzález y Berzosa consideran que "resulta difícil para el profesorado en formación detectar en ejemplos reales la existencia de situaciones tanto implícitas como explícitas de desigualdad, discriminación o violencia simbólica en base al género" (2018, p. 46). Formación que debe referirse al contexto del aula y tener una orientación práctica (Domínguez, Calvo y Vázquez, 2015; MoriñaDiez y Parrilla-Latas, 2006; Oliver-Trobat, 2009; Rivas-Borrel, 2014), por lo que debe establecerse, según Zabalza-Beraza y Zabalza-Cerdeiriña (2011) una adecuada integración entre la formación inicial y la formación permanente del profesorado. En relación con el importante avance hacia una escuela más inclusiva entienden Durán-Gisbert y Giné (2012) que "tanto la formación inicial como la formación del profesorado en servicio (...) deben enfatizar la idea de que la respuesta a la diversidad educativa del alumnado es una tarea colectiva" (2012, p. 42).

Respecto a las competencias que la normativa asigna al claustro tanto a su importancia como a la atención que a ellas les dedica el órgano de gobierno, entienden Raso-Sánchez et al. (2017) que la percepción que tiene el profesorado de los factores organizativos del centro mejora en el tiempo, según se observa al analizar los estudios realizados.

\section{Objetivos}

Los objetivos del presente trabajo se refieren a los aspectos que se indican en relación con el claustro del profesorado:

1. Examinar la formación inicial y permanente del profesorado.

2. Valorar las competencias que le asigna la normativa y la importancia que tiene para el funcionamiento del centro y la educación del alumnado.

3. Conocer el grado del cumplimiento respecto a sus competencias.

4. Analizar el tiempo de duración de sus sesiones y su frecuencia anual.

5. Investigar su importancia como órgano de participación.

6. Conocer la valoración que de este realiza el profesorado respecto a su eficacia y la atención a los problemas reales del centro.

7. Estudiar la valoración que las administraciones educativas realizan de su funcionamiento y la atención que prestan a sus acuerdos y sugerencias. 


\section{MÉTOdO}

\subsection{Muestra}

Para conocer la importancia del claustro en relación con el funcionamiento del centro se ha realizado una consulta al profesorado que imparte docencia en ESO y bachillerato de las diferentes comunidades autónomas de España. Para ello se ha elaborado un protocolo ad hoc que fue enviado por correo electrónico a una muestra de centros seleccionados al azar, en función de la población de las distintas provincias de España y de su titularidad. La muestra ha sido extraída del Registro Estatal de Centros Docentes no Universitarios del Ministerio de Educación. El cuestionario estaba acompañado de una carta en la que se señalaban los objetivos del trabajo y la importancia de la colaboración solicitada, indicando que las respuestas se recogían en una aplicación de Google e indicando que el envío de las respuestas se realizase de manera totalmente anónima.

De los 1318 docentes que han cumplimentado el protocolo propuesto, el $58,3 \%$ son profesoras, el $41,4 \%$ profesores y el ,3\% no responde respecto a su sexo. Su edad media es de 47,8 años (profesoras 46,7 y profesores 49,2, grado de significación, 000 , en adelante $\rho$ ). Su experiencia profesional media es de 19,7 años (profesoras 18,4 y profesores 21,5, $\rho, 000$ ). El 90,1 \% ejerce en centros públicos, el 9,6\% en centros privados-concertados y el ,4\% en centros privados. El 71,2 \% imparte preferentemente docencia en ESO y el $28,8 \%$ en bachillerato.

\subsection{Variables e instrumentos}

Con el presente trabajo se pretende realizar un estudio exploratorio que nos informe sobre la importancia que para el profesorado de ESO y bachillerato tiene el claustro para la educación del alumnado y el funcionamiento del centro. Las competencias analizadas del órgano de gobierno son las establecidas en el artículo 129 de la Ley Orgánica de 2/2006, de 3 de mayo de Educación.

La validez del contenido se ha analizado mediante "el juicio de expertos" (Escobar-Pérez y Cuervo-Martínez, 2008). Dichos expertos son tres inspectores 
de educación, tres directores y directoras de centros de ESO y bachillerato (dos públicos y uno privado-concertado) y cuatro profesores y profesoras de dichas enseñanzas (tres de titularidad pública y uno privada-concertada). En relación con la validez del constructo, de los 52 ítems que lo componen, la medida de adecuación muestral de Kaiser-Meyer-Olkin (KMO) tiene un valor de ,949 y la prueba de esfericidad de Bartlett un grado de significación de ,000. En el análisis factorial que se ha realizado con dichos ítems el método de extracción utilizado ha sido el Análisis de Componentes Principales y el método de rotación Varimax con Kaiser. La rotación converge en 8 iteraciones. Ocho factores explican el 70,0 \% de la varianza, con valores respectivos del 19,7, 16,9, 14,3, $4,5,4,3,4,2,3,8$ y $2,3 \%$.

Los factores se corresponden con la temática siguiente: Factor 1. " "Atención y dedicación del claustro a sus competencias". Factor 2. "Importancia de diferentes temas para la formación continua del profesorado". Factor 3. " "Importancia de las competencias del claustro para el funcionamiento general del centro". Factor 4. "Satisfacción del profesorado con el claustro e importancia de éste para la educación del alumnado". Factor 5. "Valoración de la formación inicial y permanente respecto a la participación en el claustro". Factor 6. "Participación en la selección del director y conocer las candidaturas a la dirección". Factor 7. "Valoración del funcionamiento del claustro por parte de las Administraciones educativas y atención que prestan a sus acuerdos y sugerencias". Factor $8 .^{\circ}$ "Promover por parte del claustro iniciativas en el ámbito de la investigación y experimentación y de la formación del profesorado".

Para calcular la fiabilidad de la prueba se utiliza el coeficiente de Cronbach, el cual alcanza un valor de Alpha =,96 (en la escala de 0 a 1) para el total de los 52 elementos analizados. Respecto a los tres elementos de la formación inicial y permanente la fiabilidad es de ,73. En relación con la importancia de los temas de formación (13 elementos), el coeficiente de Cronbach es ,95. La satisfacción como miembro del claustro y su importancia, con 3 elementos, tiene un coeficiente de ,76. La importancia de las competencias del claustro para la educación del alumnado y el funcionamiento del centro (14 elementos) alcanza un coeficiente de Cronbach de ,94. La importancia del claustro como órgano de participación, su eficacia y la atención que dedica a los problemas del centro ( 5 elementos) tiene un coeficiente de,87. La atención que el claustro dedica a sus competencias (14 elementos) tiene un coeficiente de Cronbach de ,95. 
TABLA 1

Coeficientes de fiabilidad de Cronbach

\begin{tabular}{|l|c|}
\hline \multicolumn{1}{|c|}{ Temas } & $\begin{array}{c}\text { Coeficiente } \\
\text { de Cronbach }\end{array}$ \\
\hline 1. Formación inicial y permanente (3 elementos) &, 73 \\
\hline 2. Importancia de temas de formación (13 elementos) &, 95 \\
\hline $\begin{array}{l}\text { 3. Satisfacción como miembro del claustro. Importancia para la educación del } \\
\text { alumnado y el funcionamiento del centro (3 elementos) }\end{array}$ &, 76 \\
\hline $\begin{array}{l}\text { 4. Importancia de las competencias del claustro para la educación del alumnado y } \\
\text { el funcionamiento del centro (14 elementos) }\end{array}$ &, 94 \\
\hline $\begin{array}{l}\text { 5. Importancia como órgano de participación. Eficacia. Atención a los problemas del } \\
\text { centro. Valoración y atención por parte de las administraciones educativas (5 elementos) }\end{array}$ &, 87 \\
\hline 6. Atención y dedicación que dedica a sus competencias (14 elementos) &, 95 \\
\hline Total (52 elementos) &, 96 \\
\hline
\end{tabular}

El cuestionario propuesto consta de los siguientes elementos:

1. Variables de clasificación: sexo, edad, experiencia docente, titularidad del centro, tamaño del centro (según número de alumnos y alumnas).

2. Formación inicial y permanente. "Formación inicial recibida en los estudios universitarios respecto a las competencias y funcionamiento del claustro", "formación inicial que reciben en la actualidad los estudiantes de profesorado respecto a las competencias y funcionamiento del claustro" y "formación permanente que el profesorado recibe de las administraciones educativas respecto a las competencias y funcionamiento del claustro".

3. Importancia de distintos temas para la formación continua del profesorado integrante del claustro: "normativa", "pedagogía-psicología, "nuevas tecnologías", "resolución de conflictos y convivencia", "gestión del cambio e innovación", "trabajo cooperativo", "metodología didáctica", "atención a las familias", "dinámica de grupos", organización y gestión de equipos docentes", "orientación escolar", "evaluación del rendimiento escolar" y "habilidades sociales".

4. Competencias del claustro para la educación del alumnado y el funcionamiento del centro y cumplimiento de las mismas por parte del claustro: "formular al equipo directivo y al consejo escolar propuestas para la elaboración de los 
proyectos del centro y de la programación general anual", "aprobar y evaluar la concreción del currículo y todos los aspectos educativos de los proyectos y de la programación general anual", "fijar los criterios referentes a la orientación, tutoría, evaluación y recuperación del alumnado", "promover iniciativas en el ámbito de la experimentación y de la investigación pedagógica", "promover iniciativas en el ámbito de la formación del profesorado del centro", "elegir a sus representantes en el consejo escolar del centro", "participar en la selección del director o directora" (en el caso de que proceda según las características del centro), "conocer las candidaturas a la dirección y los proyectos de dirección presentados por los candidatos o candidatas" (en el caso de que proceda según las características de su centro), "analizar y valorar el funcionamiento general del centro", "analizar y valorar la evolución del rendimiento escolar", "analizar y valorar los resultados de las evaluaciones internas y externas en las que participe el centro", "informar sobre las normas de organización y funcionamiento del centro", "conocer la resolución de conflictos disciplinarios y la imposición de sanciones y velar por que éstas se atengan a la norma vigente" y "proponer medidas e iniciativas que favorezcan la convivencia en el centro".

5. "Tiempo de duración media de las sesiones del claustro", "número de sesiones del claustro que se celebran anualmente en el centro", "valoración de la importancia del claustro en los centros en los que usted es o ha sido docente como órgano de participación del profesorado", "eficacia del claustro", "cómo atiende el claustro los problemas reales del centro", "cómo valoran las administraciones educativas el funcionamiento de los claustros" y “cómo atienden las administraciones educativas los acuerdos y sugerencias que realizan los claustros".

Para realizar los análisis estadísticos correspondientes se utilizan los descriptivos, diferencia de medias y análisis multivariado (utilizando el método Scheffe). Las escalas que se proponen para las distintas valoraciones tienen un recorrido de 0 a 10 puntos, siendo el 0 el menor valor y el 10 el máximo. En relación con la edad del profesorado que responde a las cuestiones planteadas se establecen tres grupos de semejantes porcentajes, el primero de ellos es el de 43 o menos años, el segundo de 44 a 52 años y el tercero de 53 o más años. Respecto a los años de experiencia docente se establecen, asimismo, tres grupos, el primero de 14 o menos años, le sigue el de 14 a 25 años y el último de 26 o más años. 


\section{Resultados}

\subsection{Formación inicial y permanente del profesorado respecto a las competencias del claustro}

La valoración media que realiza el profesorado respecto a la formación recibida en los estudios universitarios, antes de acceder a la docencia, sobre las competencias y funcionamiento del claustro es de 2,7 puntos. El 70,7 \% de las respuestas otorgan menos de cinco puntos. Valora mejor dicha formación el de los centros privados-concertados que el de los públicos (3,6 y 2,6 puntos, respectivamente, $\rho, 000)$. A este respecto no se ha solicitado en esa ocasión (aunque quizá proceda realizarlo en posteriores trabajos) al profesorado consultado la titularidad de la institución universitaria en la que ha realizado dicha formación inicial. No se encuentran diferencias en función del sexo, edad, tiempo de experiencia docente y tamaño del centro (en función del número de alumnos y alumnas).

La formación inicial que reciben en la actualidad los estudiantes de profesorado respecto a dichas competencias y funcionamiento del claustro es valorada con 4,3 puntos. En este caso tampoco se ha solicitado (quedando, asimismo, pendiente para futuros estudios) la procedencia del conocimiento que tienen sobre dicha formación. El 11,2 \% le otorga menos de cinco puntos. El grupo de mayor experiencia docente valora mejor dicha formación que el de menor experiencia ( 4,5 y 4,0 punto, $\rho, 015)$. También es mejor valorada por el profesorado de los centros privados-concertados que por el de los públicos $(4,8$ y 4,2 puntos, $\rho, 025)$. No hay diferencias según el sexo y el número de alumnos y alumnas del centro.

La valoración que profesores y profesoras consultados otorgan a la formación permanente que reciben por parte de las administraciones educativas, respecto a las competencias y funcionamiento del claustro, es de 3,8 puntos. El 8,0 \% la valora con menos de cinco puntos. No se encuentran diferencias en función de las variables analizadas. 


\subsection{Importancia de diferentes temas para la formación continua como profesor o profesora integrante del claustro}

El profesorado otorga una importante valoración a los temas que se han propuesto de formación permanente. El menor valor lo asigna a la "normativa" (7,2 puntos) y el mayor a la "resolución de conflictos y convivencia" $(8,4)$. Con ocho puntos se valora "la metodología didáctica". Entre siete y ocho puntos están valorados los restantes temas propuestos.

TABLA 2

Formación permanente

\begin{tabular}{|l|c|c|}
\hline \multicolumn{1}{|c|}{ Temas de formación permanente } & Media & Desviación \\
\hline 1. Normativa & 7,2 & 2,2 \\
\hline 2. Pedagogía-psicología & 7,8 & 2,3 \\
\hline 3. Nuevas tecnologías & 7,9 & 1,9 \\
\hline 4. Resolución de conflictos y convivencia & 8,4 & 1,9 \\
\hline 5. Gestión del cambio e innovación & 7,5 & 2,0 \\
\hline 6. Trabajo cooperativo & 7,6 & 2,1 \\
\hline 7. Metodología didáctica & 8,0 & 1,9 \\
\hline 8. Atención a las familias & 7,7 & 1,9 \\
\hline 9. Dinámica de grupos & 7,5 & 2,1 \\
\hline 10. Organización y gestión de equipos docentes & 7,4 & 2,2 \\
\hline 11. Orientación escolar & 7,4 & 2,1 \\
\hline 12. Evaluación del rendimiento escolar & 7,6 & 2,0 \\
\hline 13. Habilidades sociales & 7,9 & 2,0 \\
\hline
\end{tabular}

4.3 Importancia de cada una de las competencias del claustro para la educación del alumnado y el funcionamiento del centro. Atención que el claustro presta a sus competencias

El profesorado otorga una notable valoración a la importancia que tienen las 14 competencias del claustro para la educación del alumnado y el fun- 
cionamiento del centro. El recorrido va desde el menor valor de 7,5 puntos, referido a "elegir a sus representantes en el consejo escolar del centro", hasta el máximo valor de 8,6 para la competencia de "proponer medidas e iniciativas que favorezcan la convivencia en el centro".

El recorrido de las valoraciones que el órgano de gobierno presta a sus competencias va del menor valor de "promover iniciativas en el ámbito de la experimentación y de la investigación pedagógica", con 6,0 puntos, al máximo de "informar las normas de organización y funcionamiento del centro", con 7,3 puntos.

TABLA 3

Importancia que tiene cada una de las competencias del claustro para la educación del alumnado y el funcionamiento del centro. Atención que dedica a sus competencias

(1) Importancia de las competencias del claustro para la educación del alumnado y el funcionamiento del centro.

(2) Atención que dedica a sus competencias.

\begin{tabular}{|c|c|c|c|c|c|}
\hline \multicolumn{1}{|c|}{ Competencias del claustro } & \multicolumn{2}{|c|}{ (1) } & \multicolumn{2}{|c|}{ (2) } & Correlación \\
\cline { 2 - 6 } & Media & Desviación & Media & Desviación & \\
\hline $\begin{array}{l}\text { 1. Formular al equipo directivo y } \\
\text { al Consejo Escolar propuestas para } \\
\text { la elaboración de los proyectos del } \\
\text { centro y de la programación general } \\
\text { anual }\end{array}$ & 7,8 & 1,8 & 6,3 & 2,3 &, $360^{* *}$ \\
\hline $\begin{array}{l}\text { 2. Aprobar y evaluar la concreción } \\
\text { del currículo y todos los aspectos } \\
\text { educativos de los proyectos y de la } \\
\text { programación general anual }\end{array}$ & 7,6 & 1,9 & 6,4 & 2,3 &, $415^{* *}$ \\
\hline $\begin{array}{l}\text { 3. Fijar los criterios referentes a } \\
\text { la orientación, tutoría, evaluación } \\
\text { y recuperación de los alumnos y } \\
\text { alumnas }\end{array}$ & 7,9 & 1,9 & 6,3 & 2,4 &, $386^{* *}$ \\
\hline $\begin{array}{l}\text { 4. Promover iniciativas en el ámbito } \\
\text { de la experimentación y de la inves- } \\
\text { tigación pedagógica }\end{array}$ & 7,7 & 2,0 & 6,0 & 2,5 &, $371^{* *}$ \\
\hline $\begin{array}{l}\text { 5. Promover iniciativas en el ámbito } \\
\text { de la formación del profesorado del } \\
\text { centro }\end{array}$ & 7,8 & 1,9 & 6,4 & 2,4 &, $359^{* *}$ \\
\hline
\end{tabular}


(1) Importancia de las competencias del claustro para la educación del alumnado y el funcionamiento del centro.

(2) Atención que dedica a sus competencias.

\begin{tabular}{|l|c|c|c|c|c|}
\hline \multicolumn{1}{|c|}{ Competencias del claustro } & \multicolumn{2}{|c|}{ (1) } & \multicolumn{2}{|c|}{ (2) } & Correlación \\
\cline { 2 - 6 } & Media & Desviación & Media & Desviación & \\
\hline $\begin{array}{l}\text { 6. Elegir a sus representantes en el } \\
\text { Consejo Escolar }\end{array}$ & 7,5 & 2,2 & 6,9 & 2,3 &, $521^{* *}$ \\
\hline $\begin{array}{l}\text { 7. Participar en la selección del di- } \\
\text { rector o directora }\end{array}$ & 7,9 & 2,4 & 6,1 & 3,0 &, $418^{* *}$ \\
\hline $\begin{array}{l}\text { 8. Conocer las candidaturas a la di- } \\
\text { rección y los proyectos de dirección }\end{array}$ & 8,1 & 2,2 & 6,4 & 2,9 &, $423^{* *}$ \\
\hline $\begin{array}{l}\text { 9. Analizar y valorar el funciona- } \\
\text { miento general del centro }\end{array}$ & 8,4 & 1,7 & 7,0 & 2,2 &, $359^{* *}$ \\
\hline $\begin{array}{l}\text { 10. Analizar y valorar la evolución } \\
\text { del rendimiento escolar }\end{array}$ & 8,2 & 1,7 & 7,2 & 2,1 &, $388^{* *}$ \\
\hline $\begin{array}{l}\text { 11. Analizary valorar los resultados } \\
\text { de las evaluaciones internas y exter- } \\
\text { nas del centro }\end{array}$ & 7,9 & 1,9 & 6,9 & 2,2 &, $402^{* *}$ \\
\hline $\begin{array}{l}\text { 12. Informar las normas de organi- } \\
\text { zación y funcionamiento del centro }\end{array}$ & 8,5 & 1,5 & 7,3 & 2,0 &, $408^{* *}$ \\
\hline $\begin{array}{l}\text { 13. Conocer la resolución de con- } \\
\text { flictos disciplinarios y la imposición } \\
\text { de sanciones y velar por que estas } \\
\text { se atengan a la ley vigente }\end{array}$ & 8,1 & 1,8 & 6,9 & 2,3 &, $434^{* *}$ \\
\hline $\begin{array}{l}\text { 14. Proponer medidas e iniciativas } \\
\text { que favorezcan la convivencia en } \\
\text { el centro }\end{array}$ & 8,6 & 1,5 & 7,1 & 2,2 &, $373^{* *}$ \\
\hline
\end{tabular}

${ }^{* *}$ La correlación es significativa al nivel ,01 (bilateral)

Las profesoras valoran mejor que los profesores la importancia de todas las competencias del claustro para la educación del alumnado y el funcionamiento del centro, así como la atención que dedica a sus competencias, con excepción de las competencias referentes a la elección de la dirección y conocimiento de candidaturas y proyectos presentados, el funcionamiento del centro y sus normas de organización, entre las que no se encuentran diferencias.

En las valoraciones que se realizan respecto a la edad y el tiempo de experiencia docente no se establecen diferencias. 
Se consideran más importantes para los docentes de los centros privadosconcertados las competencias de "fijar los criterios referentes a la orientación, tutoría, evaluación y recuperación de los alumnos y alumnas", "promover iniciativas en el ámbito de la experimentación y de la investigación pedagógica", "promover iniciativas en el ámbito de la formación del profesorado del centro" y "analizar y valorar los resultados de las evaluaciones internas y externas en las que participe el centro". Por el contrario, el profesorado de los centros públicos valora mejor las competencias de "elegir sus representantes en el consejo escolar del centro", "participar en la selección del director o directora" y "conocer las candidaturas a la dirección y los proyectos de dirección presentados por los candidatos".

Es mejor valorada por parte del profesorado de los centros privados-concertados la atención que el claustro dedica a las competencias de "fijar los criterios referentes a la orientación, tutoría, evaluación y recuperación de los alumnos y alumnas", "promover iniciativas en el ámbito de la experimentación y de la investigación pedagógica", "promover iniciativas en el ámbito de la formación del profesorado del centro", "analizar y valorar los resultados de las evaluaciones internas y externas en las que participe el centro", "informar las normas de organización y funcionamiento del centro", "conocer la resolución de conflictos disciplinarios y la imposición de sanciones y velar por que éstas se atengan a la normativa vigente" y "proponer medidas e iniciativas que favorezcan la convivencia en el centro".

El de los centros públicos valora mejor la atención del claustro a las competencias de "elegir sus representantes en el consejo escolar del centro", "participar en la selección del director o directora" y "conocer las candidaturas a la dirección y los proyectos de dirección presentados por los candidatos o candidatas".

\subsection{Análisis de diversos aspectos del claustro}

\subsubsection{Importancia del claustro en la educación del alumnado del centro}

El profesorado de ESO y de Bachillerato valora con 7,5 puntos de media la importancia que tiene el claustro en la educación del alumnado (desviación 
2,1). El 9,4 \% le otorga menos de cinco puntos. Es mejor valorada por las profesoras, por el profesorado de menor tiempo de experiencia docente y por el de los centros privados-concertados. No se encuentran diferencias significativas según la edad y tamaño del centro.

TABLA 4

Importancia del claustro para la educación del alumnado del centro

\begin{tabular}{|c|c|c|c|c|}
\hline \multirow{2}{*}{$\begin{array}{l}\text { Importancia del claustro para la edu- } \\
\text { cación del alumnado del centro }\end{array}$} & Media & Desviación & Mediana & Moda \\
\hline & 7,5 & 2,1 & 8,0 & 8,0 \\
\hline \multicolumn{2}{|l|}{ 1. Sexo } & \multicolumn{3}{|c|}{$\rho$} \\
\hline Grupos & Media & \multicolumn{2}{|c|}{ Grupo 1} & Grupo 2 \\
\hline Profesoras & 7,8 & & &, 000 \\
\hline Profesores & 7,2 & & & \\
\hline \multicolumn{2}{|l|}{ 2. Tiempo de experiencia docente } & \multicolumn{3}{|c|}{$\rho$} \\
\hline Grupos & Media & Grupo 1 & Grupo 2 & Grupo 3 \\
\hline Hasta 14 años & 7,7 & & &, 023 \\
\hline De 15 a 25 años & 7,6 & & &, 031 \\
\hline 26 o más años & 7,3 & & & \\
\hline \multicolumn{2}{|l|}{ 3. Titularidad del centro } & \multicolumn{3}{|c|}{$\rho$} \\
\hline Grupos & Media & \multicolumn{2}{|c|}{ Grupo 1} & Grupo 2 \\
\hline Públicos & 7,5 & & &, 002 \\
\hline Privados-concertados & 8,1 & & & \\
\hline
\end{tabular}

\subsubsection{Importancia del claustro para el buen funcionamiento del centro}

El profesorado otorga 8,4 puntos a la importancia del claustro para el buen funcionamiento del centro. Solo el $4 \%$ lo valora con menos de cinco puntos. Dicha importancia es mejor para las profesoras y para el profesorado de los centros privados-concertados. No se establecen diferencias en función de los grupos de edad, experiencia docente y tamaño del centro. 
TABLA 5

Importancia del claustro para el buen funcionamiento del centro

\begin{tabular}{|l|c|c|c|c|}
\hline \multirow{2}{*}{$\begin{array}{l}\text { Importancia del claustro para el buen } \\
\text { funcionamiento del centro }\end{array}$} & Media & Desviación & Mediana & Moda \\
\cline { 2 - 5 } & 8,4 & 1,7 & 9,0 & 10,0 \\
\hline 1. Sexo & & \multicolumn{2}{|c|}{$\rho$} \\
\hline Grupos & Media & Grupo 1 & Grupo 2 \\
\hline Profesoras & 8,6 & & \multicolumn{2}{|c|}{$\rho$} \\
\hline Profesores & 8,2 & & \multicolumn{2}{|c|}{ Grupo 2 } \\
\hline 2. Titularidad del centro & & & Grupo 1 & \\
\hline Grupos & Media & Gru \\
\hline Públicos & 8,4 & & \\
\hline Privados-concertados & 8,8 & & \\
\hline
\end{tabular}

4.4.3 Satisfacción como miembro de los claustros de los centros en los que ha impartido docencia

El profesorado consultado otorga un valor de 6,7 puntos a su satisfacción como miembro del claustro. El 11,8 \% la valora con menos de cinco puntos. El que ejerce en centros privados-concertados otorgan mayor puntuación que el de los públicos.

\subsubsection{Duración media de los claustros que se celebran en su centro y número de sesiones anuales}

Las sesiones del claustro tienen una duración media de 88,4 minutos, con una desviación de 31,7 minutos. No se encuentran diferencias en función de las distintas variables analizadas.

Se reúnen una media de 6,6 veces al año. Los privados-concertados, 10,3 sesiones anuales y los públicos, $6,2(\rho, 000)$. No se encuentran diferencias en función del tamaño del centro. 
4.4.5 Importancia del claustro como órgano de participación del profesorado. Eficacia del claustro. Atención del claustro a los problemas reales del centro

Se valora con 7,0 puntos (desviación 2,2), la importancia del claustro como órgano de participación del profesorado. El 11,7 \% le otorga menos de cinco puntos. No se encuentran diferencias en función de las variables estudiadas.

La eficacia del claustro se valora con 6,3 puntos. El 17,9 \% le otorga menos de cinco puntos. Es más eficaz el claustro para las profesoras y para el profesorado de los centros privados-concertados. No se encuentran diferencias según la edad, tiempo de experiencia docente y tamaño del centro.

Se valora con 6,4 puntos (desviación 2,0) cómo el claustro atiende los problemas reales del centro, otorgando el 16,0 \% menos de cinco puntos. El de los centros privados-concertados le da una mayor valoración que el de los públicos ( 7,0 y 6,4 puntos, respectivamente, $\rho, 001)$. No se encuentran diferencias en función de las restantes variables analizadas.

TABLA 6

Eficacia del claustro en los centros en los que usted es o ha sido docente

\begin{tabular}{|l|c|c|c|c|}
\hline \multirow{2}{*}{$\begin{array}{l}\text { Eficacia del claustro en los centros } \\
\text { en los que usted es o ha sido docente }\end{array}$} & Media & Desviación & Mediana & Moda \\
\cline { 2 - 5 } & 6,3 & 2,1 & 7,0 & 7,0 \\
\hline 1. Sexo & Media & Grupo 1 & Grupo 2 \\
\hline Grupos & 6,5 & & \multicolumn{2}{|c|}{$\rho$} \\
\hline Profesoras & 6,1 & & \multicolumn{2}{|c|}{} \\
\hline Profesores & & & Grupo 2 \\
\hline 2. Titularidad del centro & Media & Grupo 1 & ,018 \\
\hline Grupos & 6,3 & & \\
\hline Públicos & 6,8 & & \\
\hline Privados-concertados & & &
\end{tabular}


4.4.6 Valoración por parte de las administraciones educativas del funcionamiento de los claustros y su atención a los acuerdos y sugerencias que realizan

Se otorga 4,7 puntos a la opinión que se tiene sobre la valoración que las administraciones educativas realizan del funcionamiento de los claustros. El $38,9 \%$ le proporciona menos de cinco puntos. Realiza mejor valoración el profesorado de los centros privados-concertados y el grupo de menor edad respecto al de mayor edad. No se encuentran diferencias en función de las restantes variables.

TABLA 7

Valoración por parte de las administraciones educativas del funcionamiento de los claustros

\begin{tabular}{|c|c|c|c|c|}
\hline \multirow{2}{*}{$\begin{array}{l}\text { Valoración por parte de las adminis- } \\
\text { traciones educativas del funciona- } \\
\text { miento de los claustros }\end{array}$} & Media & Desviación & Mediana & Moda \\
\hline & 4,7 & 2,4 & 5,0 & 5,0 \\
\hline \multicolumn{2}{|l|}{ 1. Titularidad del centro } & \multicolumn{3}{|c|}{$\rho$} \\
\hline Grupos & Media & \multicolumn{2}{|c|}{ Grupo 1} & Grupo 2 \\
\hline Públicos & 4,6 & & &, 001 \\
\hline Privados-concertados & 5,4 & & & \\
\hline \multicolumn{2}{|l|}{ 2. Edad } & \multicolumn{3}{|c|}{$\rho$} \\
\hline Grupos & Media & Grupo 1 & Grupo 2 & Grupo 3 \\
\hline Hasta 43 años & 5,0 & & &, 034 \\
\hline De 44 a 52 años & 4,6 & & & \\
\hline 53 o más años & 4,5 & & & \\
\hline
\end{tabular}

Se valora con 4,0 puntos cómo atienden las administraciones educativas los acuerdos y sugerencias que realizan los claustros, con menos de cinco puntos el 53,6 \%. Es mejor valorada por las profesoras, el profesorado de los centros privados-concertados, el grupo de menor edad y el de menor experiencia docente. 
TABLA 8

Atención por parte de las administraciones educativas a los acuerdos y sugerencias que realizan los claustros

\begin{tabular}{|c|c|c|c|c|}
\hline \multirow{2}{*}{$\begin{array}{l}\text { Atención por parte de las adminis- } \\
\text { traciones educativas a los acuerdos y } \\
\text { sugerencias que realizan los claustros }\end{array}$} & Media & Desviación & Mediana & Moda \\
\hline & 4,0 & 2,4 & 4,0 & 5,0 \\
\hline \multicolumn{2}{|l|}{ 1. Sexo } & \multicolumn{3}{|c|}{$\rho$} \\
\hline Grupos & Media & \multicolumn{2}{|c|}{ Grupo 1} & Grupo 2 \\
\hline Profesoras & 4,1 & & &, 001 \\
\hline Profesores & 3,7 & & & \\
\hline \multicolumn{2}{|l|}{ 2. Titularidad del centro } & \multicolumn{3}{|c|}{$\rho$} \\
\hline Grupos & Media & \multicolumn{2}{|c|}{ Grupo 1} & Grupo 2 \\
\hline Públicos & 3,9 & & &, 000 \\
\hline Privados-concertados & 4,7 & & & \\
\hline \multicolumn{2}{|l|}{ 3. Edad } & \multicolumn{3}{|c|}{$\rho$} \\
\hline Grupos & Media & Grupo 1 & Grupo 2 & Grupo 3 \\
\hline Hasta 43 años & 4,3 & &, 005 & 019 \\
\hline De 44 a 52 años & 3,7 & & & \\
\hline 53 o más años & 3,8 & & & \\
\hline \multicolumn{2}{|l|}{ 4. Tiempo de experiencia docente } & \multicolumn{3}{|c|}{$\rho$} \\
\hline Grupos & Media & Grupo 1 & Grupo 2 & Grupo 3 \\
\hline Hasta 14 años & 4,2 & & &, 040 \\
\hline De 15 a 25 años & 3,9 & & & \\
\hline 26 o más años & 3,7 & \multicolumn{3}{|c|}{$\rho$} \\
\hline
\end{tabular}

\section{DisCUSIÓN Y CONCLUSIONES}

El claustro y el consejo escolar son los órganos de participación de los centros educativos; el primero es específico del profesorado y el segundo, de participación de la comunidad educativa. Es, por tanto, un órgano de gran 
importancia en los centros de ESO y Bachillerato, aunque no se ha estudiado con frecuencia en la investigación educativa, ya que esta se centra con mayor asiduidad, quizá por su novedad e importancia socioeducativa, en el consejo escolar. No obstante lo anterior, existe una especial atención a su órgano específico de gobierno por parte del profesorado en su debate y quehacer diario, aunque en demasiadas ocasiones llevado a cabo con un carácter excesivamente corporativista y en contraposición y competencia con el consejo escolar, al que en ocasiones se considera como competidor más que como órgano de participación complementario y de representación de toda la comunidad educativa.

Se entiende con Ordoñez-Sierra (2008) que debe mejorarse tanto la formación inicial como permanente del profesorado de ESO y bachillerato en relación con su participación en el claustro, a la vez que, tal como señalan ZabalzaBeraza y Zabalza-Cerdeiriña (2011), es necesaria una adecuada integración de dichas etapas de formación. Se considera que dicho tema debe incluirse tanto en la formación inicial como en la permanente del profesorado, al relacionarse la calidad de la educación con la formación del profesorado (Barber y Mourshed, 2007; Tiana-Ferrer, 2011; OCDE, 2014; Rivas-Borrel, 2014; Sanz-Ponce et al. 2015). La participación en los centros no debe entenderse como un mero formalismo (Moliner-García et al., 2016) ya que se considera necesaria para el desarrollo del alumnado y el éxito escolar (Andrés-Cabello y Giró-Miranda, 2016; Bolívar, 2006) y que sin dicha participación del profesorado en los procesos de cambio educativo no existen posibilidades de que estos tengan éxito (Martín-Bris y Muñoz-Martínez, 2010).

En el presente trabajo el profesorado valora, en general, adecuadamente su satisfacción respecto a su participación en el claustro (6,7 puntos), coincidiendo con lo señalado por Raso-Sánchez et al. (2017).

Las competencias que la normativa le asigna son en ocasiones objeto de debate por parte del profesorado, entendiendo que estas son insuficientes y considerando que algunas de las que tiene el consejo escolar deberían estar atribuidas al órgano de participación del profesorado. No obstante, en el presente trabajo se entiende que dichas competencias están adecuadamente asignadas al claustro, (todas y cada una) otorgan una alta valoración a su importancia. Se puede considerar que la asignación de competencias que la normativa le otorga al órgano de gobierno es adecuada tanto para el buen funcionamiento del centro como para la calidad de la enseñanza que ofrece el centro. Y todo 
ello a pesar de las quejas que en ocasiones se manifiestan entre el profesorado. Otros aspectos adecuadamente valorados se refieren al claustro como órgano de participación, su eficacia en el centro y a cómo atiende a sus problemas reales.

El profesorado consultado considera que la formación inicial que ha recibido en su etapa universitaria respecto a este órgano de gobierno no ha sido adecua$\mathrm{da}$, siendo mejor valorada por el que ejerce en centros privados-concertados. Entendiendo, asimismo, que puede mejorarse la formación que al respecto reciben por parte de las administraciones educativas.

Los temas de formación que se han propuesto se valoran todos ellos como adecuados, en un recorrido del menor valor de 7,2 puntos al mayor de 8,4. Por otro lado, también se valoran de manera adecuada la importancia de las competencias (de un valor mínimo de 7,5 puntos a un máximo de 8,6). Se otorga menor valoración a la atención que el órgano de gobierno dedica a dichas competencias. También se da una adecuada valoración a su importancia para la educación del alumnado y para el buen funcionamiento del centro. Al grado de satisfacción como miembros del claustro le otorga el profesorado consultado 6,7 puntos, por lo que considera a este como un adecuado órgano de participación del profesorado. A la eficacia y atención que presta a los problemas reales del centro se le otorga una valoración de 6,5 puntos. La duración media de sus sesiones es de 88,4 minutos y se reúnen 6,6 veces durante el curso.

Se ha observado que las competencias asignadas por la normativa al órgano de gobierno del profesorado son adecuadas, según criterio de sus componentes; no obstante, procede que dichos órganos atiendan y ejecuten dichas competencias de manera más adecuada. Entendemos que, dada su importancia, convendría seguir analizando el claustro en cuanto a su relación con el funcionamiento del centro y la calidad de la enseñanza que ofrece a su alumnado. En la formación inicial del profesorado, en la etapa previa a su trabajo docente, debería estudiarse con mayor profundidad el órgano de gobierno indicado. De igual manera cabría mejorar los programas de formación permanente del profesorado. En próximos trabajos sería de interés conocer la titularidad de los centros universitarios en los que el profesorado ha realizado sus estudios toda vez que tanto en lo que se refiere a la formación inicial como a la permanente son mejor valoradas por el profesorado que ejerce en centros privados-concertados. Se entiende, por otro lado, que las administraciones educativas podrían mejorar la valoración que realizan del funcionamiento del órgano de gobierno 
específico del profesorado, así como la atención que prestan a los acuerdos y sugerencias que estos realizan en su actividad. En este sentido, una mejor y mayor comprensión de sus competencias ayudaría a evitar los desencuentros que en ocasiones se producen con las establecidas para el consejo escolar y centrarían más el trabajo específico de cada uno de los órganos de gobierno, a la vez que enmarcaría adecuadamente las competencias de la dirección del centro como presidente o presidenta del órgano de gobierno.

\section{BibliografíA}

Andrés-CABello, S. y Giró-Miranda, J. (2016). El papel y la representación del profesorado en la participación de las familias en la escuela. Revista Electrónica Interuniversitaria de Formación del Profesorado 19(1), 61-71. DOI: http://dx.doi.org/10.6018/reifop.19.1.24546

BARBER, M. y Mourshed, M. (2007). How the world's best performing school systems come out on top. Edit: McKinsey \& Company Report. Recuperado de: https://n9.cl/rg529

Bolívar, A. (2006). Familia y escuela: dos mundos llamados a trabajar en común. Revista de Educación 339, 119-146. Recuperado de: https://n9.cl/wddq

Constitución Española de 1978. Recuperado de: https://n9.cl/om7zy

Domínguez, J., Calvo, J. y VÁzquez, C. (2015). Evaluación de la formación permanente del profesorado: Enfoque de Resultados. Revista de Estudios e Investigación en Psicología y Educación 2(1), 11-18. Recuperado de: https:// revistas.udc.es/index.php/reipe/article/download/reipe.2015.2.1.72/pdf_1

DurAn-Gisbert, D. y Giné-Giné, C. (2017). La formación permanente del profesorado para avanzar hacia la educación inclusiva. Edetania. Estudios y Propuestas Socioeducativas 41, 31-44. Recuperado de: https://revistas. ucv.es/index.php/Edetania/article/view/260

Escobar-Pérez, J. y Cuervo-Martínez, A. (2008). Validez del contenido y juicio de expertos. Avances en Medición 6, 27-36. Recuperado de: https:// n9.cl/6xmf

Ley Orgánica de 2/2006, de 3 de mayo de Educación. BOE 4/5/2006. Recuperado de: https://www.boe.es/buscar/pdf/2006/BOE-A-2006-7899-consolidado.pdf 
Martín-Bris, M. y Muñoz-Martínez, Y. (2010). La participación del profesorado en un proceso de mejora en el marco de "Una escuela para todos". REICE: Revista Electrónica Iberoamericana sobre Calidad, Eficacia y Cambio en Educación 8(3), 120-138. Recuperado de: https://dialnet.unirioja.es/servlet/autorExterno/BNE/951511

Moliner, O., Traver, J. A., Ruiz, M. P. y Segarra, T. (2016). Estrategias que inciden en los procesos de democratización de la escuela. Una aproximación teórica. Revista Electrónica de Investigación Educativa 18(2), 116-129. Recuperado de: https://redie.uabc.mx/redie/article/view/1110/1433

Moriña-Diez, A. y Parrilla-Latas, A. (2006). Criterios para la formación permanente del profesorado en el marco de la educación inclusiva. Revista de Educación 339, 517-539. Recuperado de: https://idus.us.es/xmlui/ handle/11441/66853

OCDE (2014). TALIS (Teaching and Learning International Survey) 2013. Estudio internacional de la enseñanza y el aprendizaje. Madrid: Ministerio de Educación, Cultura y Deporte. Recuperado de: https://n9.cl/cxkoj

Oliver-Trobat, M. F. (2009). Análisis de la formación permanente del profesorado institucional en la Comunidad Autónoma de las Illes Balears. II Seminario Internacional. Nuevos retos de la profesión docente. Barcelona, España. Recuperado de: https://n9.cl/6qda6

OrdoÑEZ-Sierra, R. (2008). Análisis de las opiniones mostradas por el profesorado de educación primaria sobre su grado de participación en los centros escolares de Sevilla capital. Bordón 60(2), 139-157. Recuperado de: https:// dialnet.unirioja.es/descarga/articulo/2717073.pdf

Pinedo-González, R., Arroyo-González, M. J. y Berzosa, I. (2018). Género y educación: detección de situaciones de desigualdad de género en contextos educativos. Contextos Educativos. Revista de Educación 21, 35-51. Recuperado de: http://dx.doi.org/10.18172/con.3306

Rivas-Borrel, S. y Ugarte-Artal, C. (2014). Formación docente y cultura participativa del centro educativo: claves para favorecer la participación familia-escuela. Estudios sobre Educación 27, 153-168. DOI: https://doi. org/10.15581/004.27.153-168

Raso-Sánchez, F., Sola-Martínez, T. e Hinojo-Lucena, F.J. (2017). Satisfacción del profesorado de la escuela rural de la provincia de Granada (España) respecto a la organización escolar. Bordón. Revista de Pedagogía 69(2), 
79-96. Recuperado de: https://recyt.fecyt.es/index.php/BORDON/article/ view/41372

Ros-Garrido, A. y GARCíA-RuBIo, J. (2017). La calidad en la formación del profesorado del sistema educativo y de los certificados de profesionalidad. Edetania. Estudios y Propuestas Socioeducativas 50, 101-119. Recuperado de: https://revistas.ucv.es/index.php/Edetania/article/view/21

Sanz-Ponce, J. R., Hernando-More, I. y Mula-Benavent, J. M. (2015). La percepción del profesorado de Educación Secundaria en la Comunidad Valenciana acerca de sus conocimientos profesionales. Estudios sobre Educación 29, 215-234. Recuperado de: https://dialnet.unirioja.es/descarga/ articulo/5349084.pdf

TianA-Ferrer, A. (2011). Políticas de formación del profesorado y mejora de los sistemas educativos: algunas reflexiones a partir de la experiencia española. Revista Fuentes 11, 13-27. Recuperado de: https://idus.us.es/xmlui/ handle/11441/32896

Zabalza-Beraza, M. A. y Zabalza-Cerdeiriña, M. A. (2011). La formación del profesorado de Educación Infantil. CEE Participación Educativa 16, 103-113. Recuperado de: https://reunir.unir.net/...2013_05_23_TFM_ESTUDIO_DEL_TRABAJO.pdf? sequence... 